\title{
Avaliação da qualidade dos serviços bancários online: Proposta de integração dos modelos SERVQUAL, Kano e QFD
}

\author{
Quality assessment of online banking services: An integrated model of SERVQUAL, Kano and QFD
}

\author{
Ariana Heleno Marques Santos
}

Faculdade de Economia da Universidade do Algarve, Campus de Gambelas, 8005-139 Faro, Portugal, ariana.h.santos@gmail.com

\author{
Carlos J. F. Cândido
}

Faculdade de Economia da Universidade do Algarve e CEFAGE-UAlg, Campus de Gambelas, 8005-139 Faro, Portugal, ccandido@ualg.pt

\begin{abstract}
Resumo
Este artigo centra-se no desenvolvimento teórico de um modelo integrado para a avaliação e a melhoria da qualidade dos serviços de Internet Banking (IB). Após uma revisão da literatura e uma análise crítica dos estudos já elaborados sobre o uso integrado dos modelos de Kano, SERVQUAL e Quality Function Deployment (QFD), propõe-se um modelo integrado significativamente distinto daquele que tem vindo a ser utilizado na literatura. O modelo que se apresenta visa superar as desvantagens inerentes ao modelo integrador existente, contribuindo para um melhor entendimento da "voz do cliente" e para uma melhoria da qualidade dos serviços de IB. O artigo propõe também um conjunto de proposições para investigação empírica futura.
\end{abstract}

Palavras-chave: Internet banking, qualidade, SERVQUAL, Kano, QFD, modelo integrado.

\section{Abstract}

This article focuses on the theoretical development of an integrated mode for the assessment and improvement of the quality of Internet Banking (IB) services. Based on a literature review and critical analysis of the studies on the integrated use of the Kano model, the SERVQUAL and the Quality Function Deployment (QFD), this paper suggests an integrated model that is significantly distinct from those in the extant literature. The suggested model aims to surpass inherent disadvantages of the models in the literature, to contribute to a better understanding of the "voice of the customer" and to improve the quality of the IB services. This paper also develops several propositions for future empirical investigation.

Keywords: Internet banking, service quality, SERVQUAL, Kano, QFD, integrated model.

\section{Introdução}

A sociedade tem vindo a sofrer um conjunto pronunciado de mudanças que resultaram do progresso acentuado das tecnologias da informação e da comunicação nos últimos anos. Estas tecnologias tornaram-se quase omnipresentes na vida quotidiana, sendo cada vez maior o número de pessoas que as utilizam com grande frequência. A Internet é uma das principais tecnologias, senão mesmo a principal, para a disseminação da informação e comunicação.

Para as empresas, a Internet constitui uma poderosa ferramenta de marketing, tanto como meio de comunicação com clientes, como de canal de distribuição de produtos e serviços (Brochand, Lendrevie, Rodrigues e Dionísio, 1999). A Internet permite dar a conhecer aos consumidores os produtos e serviços de uma forma mais completa que os media tradicionais, além de dar a possibilidade de se efetuarem as correspondentes transações. Relativamente aos bancos, muitas pessoas têm trocado a agência física por transações via Internet. Neste contexto, a preocupação com a qualidade da informação e dos serviços disponibilizados nos websites tem aumentado. Tornou-se importante atingir níveis de qualidade que garantam resultados positivos para os bancos, nomeadamente, através da diferenciação dos serviços, da satisfação dos consumidores e da fidelização dos clientes.

A avaliação da qualidade dos serviços bancários via Internet já foi objeto de reflexão por parte de alguns investigadores (e.g., Jayawardhena, 2004; Castillo, Montecinos e Villagran, 2007; Yu, 2008; Loonam e O'Loughlin, 2008; Liao e Cheung, 2008; Santouridis, Trivellas e Reklitis, 2009; Khurana, 2009; Akinci, Atilgan-Inan e Aksoy, 2010). No entanto, a maioria destes utiliza apenas o SERVQUAL (Parasuraman, Zeithaml and Berry, 1988,
1991) para esse fim. O presente artigo tem como objetivo o desenvolvimento teórico de um método alternativo, integrando o SERVQUAL, o modelo de Kano (Kano, Seraku, Takahashi e Tsuji, 1984) e o método QFD (Akao, 1972) num único modelo. A integração destes três instrumentos já foi estudada por vários autores (e.g., Tan e Shen, 2000; Tan e Pawitra, 2001; Pawitra e Tan, 2003; Hsu, Chang, Wang e Lin, 2007; Yesilada e Yurdakul, 2009; Hutahaean, 2009; Ardhiyani e Singgih, 2010; Rahmana, Kamil, Soemantri e Olim, 2014). Porém, os instrumentos e a forma de os integrar podem ser especialmente adaptados a cada setor. Este trabalho propõe um modelo de integração diferente e com vantagens relativamente àqueles anteriormente apresentados na literatura.

\section{Revisão da literatura}

\subsection{Qualidade e Internet Banking}

De uma maneira geral, o Internet Banking (IB) coloca à disposição dos clientes uma vasta gama de informações sobre produtos e serviços bancários e a possibilidade de realizar um extenso conjunto de operações financeiras.

O conceito de qualidade, inicialmente direcionado apenas para os produtos manufaturados, foi sendo alargado de forma a contemplar também os serviços, abrangendo mais recentemente os serviços prestados através da Internet. Oferecer serviços de qualidade através dos websites é uma estratégia fundamental para o sucesso das empresas (Zeithaml, Parasuraman e Malhotra, 2002). Segundo os mesmos autores, a entrega de um serviço de qualidade através do website é, talvez, mais importante do que o baixo preço dos produtos/serviços e da própria presença na Web. Fatores tais como o produto, a imagem e a distribuição passaram 
a ter um papel secundário nos bancos, enquanto a qualidade do serviço passou a ser essencial (Castillo et al., 2007). Parasuraman et al. (1988) definem qualidade de serviço como o resultado da comparação entre as expetativas e as perceções dos clientes em relação a um determinado serviço. Assim, os gestores devem primeiro entender como os seus clientes percebem e avaliam o serviço online, para depois estarem em condições de oferecer um serviço de qualidade superior (Zeithaml et al., 2002).

Várias vantagens têm sido apontadas como justificação para o crescimento do IB. Por exemplo, os clientes obtêm preços mais competitivos e podem realizar as operações financeiras sem restrições de horário (Jayawardhena, 2004). Angelakopoulos e Mihiotis (2011) referem que os clientes não precisam de perder tempo nas filas das agências, pois, podem realizar as suas transações em qualquer lugar e têm à sua disposição um serviço de apoio 24 horas por dia, sete dias por semana. Para os bancos, o IB permite oferecer produtos inovadores e conquistar novos clientes (Sullivan, 2000). Segundo Angelakopoulos e Mihiotis (2011), o IB permite oferecer serviços em zonas onde os bancos não têm agências físicas; permite realizar transações com um custo menor; permite aos funcionários bancários das agências ocupar o seu tempo de trabalho a promover os produtos/serviços junto dos clientes, em vez de efetuarem transações; permite reduzir as filas nas agências, o que faz com que os funcionários se sintam menos sobrecarregados; e permite obter informações sobre os clientes, tais como preferências e tendências de utilização. Além destas vantagens, os clientes do IB são ainda mais rentáveis, utilizam mais produtos e mantêm saldos superiores aos dos clientes das agências (Hitt e Frei, 2002).

Reconhecendo estas vantagens, os bancos estão cada vez mais interessados e preocupados com o IB e estão a procurar métodos para fornecer um serviço de alta qualidade que cumpra rigorosamente as exigências dos clientes (Yu, 2008).

\subsection{O modelo SERVQUAL}

O SERVQUAL é um instrumento que permite avaliar a qualidade dos serviços numa grande variedade de setores de atividade (Parasuraman et al., 1988). Concretamente, o modelo serve para identificar os pontos fortes e os pontos fracos de um determinado serviço (Cândido, 2003; Yesilada e Yurdakul, 2009) em termos de 5 dimensões da qualidade: tangíveis, fiabilidade, atendimento, garantia e empatia (Parasuraman et al., 1988). A dimensão tangíveis diz respeito às instalações, equipamentos e aparência do pessoal. A fiabilidade consiste na capacidade para executar o serviço prometido de forma confiável e precisa. A dimensão atendimento corresponde à vontade de ajudar os clientes e fornecer um serviço rápido. A garantia é o conhecimento e cortesia do pessoal e a sua capacidade de inspirar confiança e segurança. Por último, a empatia é o cuidado e atenção personalizada que a empresa disponibiliza aos seus clientes (Parasuraman et al., 1988).

Estas cinco dimensões são desagregadas em 22 itens (atributos), que compõem um questionário a entregar aos clientes (Parasuraman et al., 1988). O questionário divide-se habitualmente em duas partes: a primeira parte, composta por 22 itens, tem como objetivo avaliar as expetativas dos clientes. A segunda parte, constituída pelos mesmos 22 itens, mas com ligeiras alterações na sua redação, pretende avaliar a perceção dos clientes (Parasuraman et al., 1991). A extensão das diferenças entre as expetativas e as perceções permite determinar o nível de qualidade do serviço prestado (Grönroos, 2007).

De acordo com Parasuraman et al. (1988), o SERVQUAL é um bom instrumento para compreender as expetativas e as perceções dos clientes. O modelo serve de apoio para formular estratégias e táticas (Tan e Pawitra, 2001; Cândido e Morris, 2000, 2001; Cândido, 2005). Contudo, vários autores apontam algumas limitações ao modelo. Uma vez que as expetativas e as perceções são avaliadas pelo mesmo indivíduo e num único momento após a realização do serviço, o inquirido acaba por responder a todas as questões com base na sua experiência (Carman, 1990). Assim, as respostas sobre o serviço desejado possuem pouco valor. Jayawardhena (2004) aponta também como limitação o facto de não se conseguir saber quais são os atributos mais relevantes para a satisfação dos clientes, isto é, o SERVQUAL pressupõe um nível de importância idêntico para todos os atributos da qualidade. Por fim, Tan e Pawitra (2001) destacam que o modelo SERVQUAL não está concebido de molde a indicar soluções para as lacunas encontradas, entre perceções e expetativas.

\subsection{0 modelo de Kano}

O modelo de Kano é uma metodologia que visa categorizar os atributos dos produtos e dos serviços com base na sua capacidade para satisfazer as necessidades dos clientes (Ardhiyani e Singgih, 2010). Segundo Löfgren e Witell (2005), o modelo distingue cinco categorias de atributos:

(1) Atributos atrativos: se estiverem presentes no produto/serviço será maior a satisfação do cliente. Se estiverem ausentes, não causam insatisfação (Sauerwein, Bailom, Matzler e Hinterhuber, 1996);

(2) Atributos unidimensionais: quanto maior o grau de desempenho do atributo, maior será a satisfação do cliente (Tan e Pawitra, 2001). Se estes atributos não estiverem presentes no serviço, causam insatisfação (Yesilada e Yurdakul, 2009);

(3) Atributos obrigatórios: são os requisitos básicos de um serviço (Yesilada e Yurdakul, 2009). Se tiverem um baixo desempenho no serviço, o cliente sente-se insatisfeito, se o desempenho for alto, não aumenta a satisfação (Tan e Pawitra, 2001);

(4) Atributos indiferentes: não resultam em satisfação ou insatisfação do cliente (Löfgren e Witell, 2005);

(5) Atributos reversos: são aqueles cuja presença no serviço causam insatisfação do cliente e cuja ausência origina satisfação (Ardhiyani e Singgih, 2010).

Para categorizar os atributos dos serviços, o modelo de Kano utiliza um questionário dirigido ao cliente (Hsu et al., 2007; Madzík, 2016). O questionário incluí duas questões relativas a cada atributo, uma questão na positiva e outra na negativa (Ardhiyani e Singgih, 2010). A combinação das respostas às duas questões permite então categorizar os atributos.

Para a construção do questionário de Kano é necessário identificar previamente quais os atributos do produto/serviço (Sauerwein et al., 1996). Este modelo, ao contrário do SERVQUAL, não ajuda a identificar os atributos ou itens do produto/serviço a avaliar. $O$ 
modelo de Kano apenas classifica os atributos já identificados. Assim sendo, é vantajosa a integração deste modelo com o SERVQUAL, uma vez que as dimensões e os itens do SERVQUAL podem ser aqui empregados com vantagem. Yesilada e Yurdakul (2009) apontam ainda como desvantagem do modelo de Kano, o facto de ter sido desenvolvido para definir qualidade de serviço no contexto das expetativas dos clientes, ou seja, avalia o grau de satisfação que um atributo poderá trazer aos clientes, mas não avalia o grau de satisfação efetivo após o consumo do produto ou serviço. Utilizando o modelo de Kano em combinação com o SERVQUAL é possível colmatar estas lacunas.

\subsection{0 método Quality Function Deployment (QFD)}

A metodologia do QFD foi desenvolvida no Japão por Yoji Akao, em 1972 (Hutahaean, 2009). Akao (1990) define QFD como um sistema que converte as exigências do cliente em características técnicas do produto, em todas as fases de desenvolvimento do mesmo, a fim de garantir a sua qualidade final.

Para transferir as exigências do cliente para o processo de desenvolvimento dos produtos/serviços utilizam-se várias matrizes. Essas matrizes são combinadas para formar a "Casa da Qualidade" (Hauser e Clausing, 1988). A ideia principal subjacente à "Casa da Qualidade" é a de que os produtos devem refletir os desejos dos clientes (Yesilada e Yurdakul, 2009). A "Casa da Qualidade" inclui informações sobre os desejos dos clientes relativos a determinado produto/serviço e a correspondente resposta da empresa para satisfazer esses desejos.

O uso do QFD reduz o tempo de desenvolvimento de um produto, ajuda a garantir a satisfação do cliente e contribui para o aumento das vendas (Akao, 1990). Contudo, o QFD apresenta algumas desvantagens, entre as quais se inclui por exemplo a ambiguidade na interpretação da "voz do cliente" (Bouchereau e Rowlands, 1999). A integração dos modelos SERVQUAL e Kano no QFD tornase útil e benéfica a este nível, na medida em que conduz a uma melhor compreensão da "voz do cliente".

\subsection{Síntese dos estudos sobre a integração do SERVQUAL, Kano e QFD}

Conforme referido no ponto 1 , a integração dos três instrumentos já foi estudada por vários autores. Todos os autores citados aplicaram essa integração a um setor de atividade diferente daquele que é abordado neste artigo e trataram de forma bastante semelhante a combinação dos três instrumentos (vide Tabela 1).

Tabela 1 - Estudos sobre a integração do SERVQUAL, Kano e QFD

\begin{tabular}{|l|l|}
\hline \multicolumn{1}{|c|}{ Estudo } & \multicolumn{1}{c|}{ Setor de Atividade } \\
\hline $\begin{array}{l}\text { Rahmana, Kamil, Soemantri e } \\
\text { Olim (2014) }\end{array}$ & $\begin{array}{l}\text { Formação baseada em simulação de gestão } \\
\text { de projetos }\end{array}$ \\
\hline Ardhiyani e Singgih (2010) & Serviço postal na Indonésia \\
\hline Hutahaean (2009) & Agência de viagens em Jakarta, Indonésia \\
\hline Yesilada e Yurdakul (2009) & Hospital Universitário em Izmir, Turquia \\
\hline Hsu, Chang, Wang e Lin (2007) & Telecomunicações móveis em Taiwan \\
\hline $\begin{array}{l}\text { Pawitra e Tan (2003), Tan e } \\
\text { Pawitra (2001) }\end{array}$ & Singapura como destino turístico \\
\hline Tan e Shen (2000) & Websites em geral \\
\hline
\end{tabular}

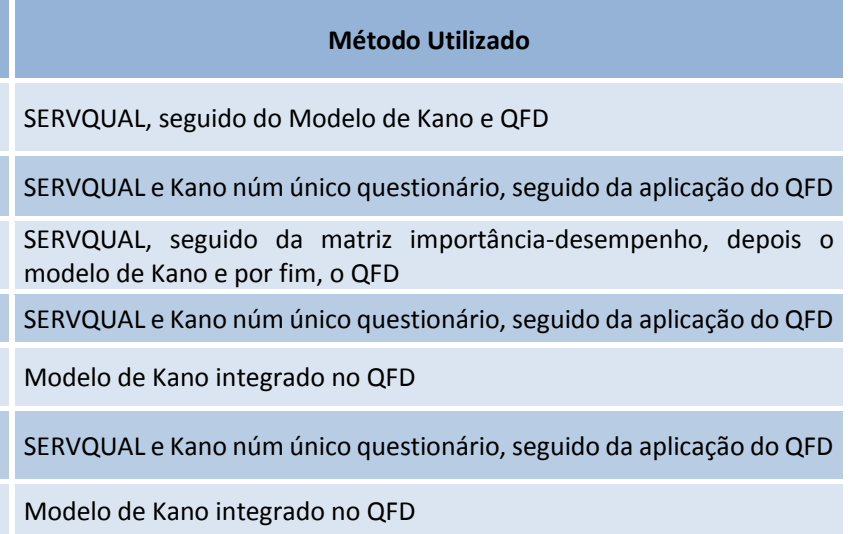

Os autores citados começaram por integrar o modelo de Kano no SERVQUAL, seguindo as etapas sugeridas por Tan e Pawitra (2001):

(1) identificar os atributos do produto/serviço,

(2) medir as expetativas e as perceções dos clientes e avaliar a importância de cada atributo e

(3) categorizar os atributos.

Relativamente à primeira etapa, alguns dos autores citados realizaram entrevistas com clientes. As entrevistas são úteis, porque levam os clientes a falar dos problemas que têm tido com o produto/serviço, mas não são suficientes para descobrir novos atributos (Sauerwein et al., 1996). Além disso, das entrevistas pode resultar uma lista demasiado grande de atributos a analisar. Por isto, devem-se usar também outros métodos, tal como Ardhiyani e Singgih (2010) fizeram, nomeadamente, uma revisão da literatura e uma entrevista à equipa de gestão da empresa. Ao realizar as entrevistas a clientes, convém ainda ter em conta os vários segmentos que a empresa pretende servir. Yesilada e Yurdakul (2009), por exemplo, tiveram em consideração os segmentos de clientes segundo as faixas etárias e os níveis de educação.
Quanto à etapa dois, a maior parte dos autores citados elaborou um questionário a partir do SERVQUAL e avaliou a satisfação dos clientes com base na diferença entre perceção e expectativa. Contudo, como foi referido neste artigo, no ponto 2.2, o SERVQUAL apresenta algumas fraquezas na forma como avalia a qualidade do serviço.

Em relação à etapa três, a categorização dos atributos foi feita com base no modelo de Kano. O questionário de Kano foi aplicado juntamente com o questionário da etapa anterior. Além das fraquezas do SERVQUAL, a junção dos modelos, tal como foi feita, parece provocar uma duplicação de esforços na avaliação das expetativas do cliente, uma vez que contempla a secção das expetativas do SERVQUAL e ainda a análise das expetativas pelo modelo de Kano.

Para concluir o processo de integração dos três modelos, que começa com as três etapas acima referidas, a maioria dos autores aplicou em seguida o modelo QFD. Mas, a aplicação do QFD só se pode revelar eficaz se a "voz do cliente" for convenientemente capturada, o que não sucede na realidade, pelo que ficou exposto. 
A Tabela 2 resume as críticas apontadas aos estudos que integraram os três instrumentos (citados na Tabela 1) e as críticas aos estudos que avaliaram a qualidade do serviço de IB (referidos no Ponto 1 deste artigo).

Tabela 2 - Resumo das críticas que o modelo a propor pretende superar

Críticas aos estudos sobre a integração do SERVQUAL, Kano e QFD (noutros setores de
atividade)

Os atributos do serviço foram obtidos maioritariamente através de entrevista com clientes, o que poderá resultar numa lista grande de atributos a avaliar e também poderá não abranger todos os atributos do serviço, nomeadamente atributos atrativos. As expetativas e as perceções dos clientes foram avaliadas no mesmo questionário e após estes terem experimentado o serviço da empresa, o que faz com que as respostas sobre o serviço desejado tenham pouco valor.

A satisfação do cliente foi medida com base na diferença entre perceção e expectativa, em conformidade com o modelo SERVQUAL.

O modelo de Kano foi aplicado conjuntamente com as duas secções do questionário SERVQUAL original, o que resulta na duplicação de esforços para averiguar as necessidades dos clientes.

Tendo em conta a forma como os modelos SERVQUAL e Kano foram integrados, os dados a dar entrada na "Casa da Qualidade" não são satisfatórios, pelo que o método QFD não consegue produzir os melhores resultados para aumentar a qualidade do serviço e a satisfação dos clientes.

\section{Proposta metodológica e proposições de pesquisa}

\subsection{Integração dos modelos SERVQUAL, Kano e QFD}

O modelo integrado aqui proposto, visa superar as desvantagens inerentes a cada um dos instrumentos e contribuir para um melhor entendimento da "voz do cliente". A satisfação total do cliente é o objetivo final do QFD, logo, é necessário ouvir e compreender o que os clientes desejam (Ardhiyani e Singgih, 2010).

O primeiro passo para avaliar a qualidade do serviço de IB é construir uma lista de atributos de websites bancários (Kuo, Yuo e Lu, 2014; Song, 2016). Para tal, este artigo propõe que se faça uma revisão da literatura e dois focus groups, um composto por clientes do serviço bancário e outro composto por gestores de IB. A conjugação destes dois focus groups permite reduzir a dificuldade dos gestores em perceberem as necessidades dos clientes, um problema identificado por Parasuraman, Zeithaml e Berry (1985). Também com o objetivo de mitigar este problema, tem sido sugerida, em alternativa, a realização de entrevistas com clientes, com especialistas e com gestores, o uso do brainstorming e, até, do método Delphi (e.g., Ardhiyani e Singgih, 2010; Kuo et al., 2014; Nelson-Rowe, Kraft e Varney, 2016:25; Madzík, 2016).

Convém salientar que não há qualquer inconveniente em consultar outros stakeholders (e.g., especialistas). Nem há inconveniente em que alguns clientes incluídos nos focus groups tenham já anteriormente experimentado o serviço, porque, nesta fase, apenas se pretende identificar as necessidades para determinar os atributos do website. As expetativas e perceções serão avaliadas a posteriori e em fases distintas desta.

O segundo passo consiste em conhecer as expetativas do cliente em relação ao serviço. Para esse efeito, propõe-se a utilização do modelo de Kano e do SERVQUAL, nesta ordem, o que representa uma clara inversão relativamente ao que tem sido feito em
Críticas aos estudos que utilizaram apenas o SERVQUAL para medirem a qualidade dos serviços de IB

As cinco dimensões originais do SERVQUAL não estão todas incluídas no instrumento de avaliação.

As dimensões acrescentadas podem não ser totalmente novas, poderão estar já incluídas nas dimensões originais do SERVQUAL.

Na maior parte dos estudos as expetativas dos clientes não foram identificadas. estudos anteriores. O objetivo é substituir a secção das expetativas do SERVQUAL pelo modelo de Kano. Repare-se que o modelo de Kano foi desenvolvido a fim de definir qualidade de serviço no contexto das expetativas do cliente (Yesilada e Yurdakul, 2009). Desta forma, é possível ultrapassar as críticas apontadas na Tabela 2 à forma como o SERVQUAL vinha sendo empregue nos estudos anteriores, designadamente, a má qualidade da informação fornecida ao modelo QFD, a duplicação de esforços na medição das expetativas e a avaliação das expetativas no mesmo questionário em que se avaliavam as perceções. A utilização do modelo de Kano permite ainda evitar o pressuposto do SERVQUAL de que todos os atributos são igualmente importantes (Hutahaean, 2009) e permite também identificar os atributos atrativos.

No terceiro passo, avalia-se a satisfação dos clientes que já utilizaram o serviço, através das suas perceções, aplicando-se para o efeito apenas a segunda parte do SERVQUAL, cuja finalidade é precisamente a de avaliar perceções (Parasuraman et al., 1988, 1991). O grau de satisfação dos clientes, apurado desta forma, pode ser cruzado com a categorização dos atributos que foi efetuada nas etapas anteriores, permitindo assim aos bancos o estabelecimento de prioridades para o desenvolvimento do seu serviço de IB e a realização de melhorias nos serviços.

O resultado final da avaliação da qualidade torna-se assim mais rigoroso, por não enfermar de um conjunto de desvantagens que já foram criticadas anteriormente (Tabela 2), contribuindo também para uma melhor audição do cliente.

Após ouvir e compreender o cliente, o quarto passo consiste em criar um plano de ação que, com o auxílio do QFD, vise a melhoria do serviço prestado. A Figura 1 ilustra esta proposta metodológica, em quatro passos, para a medição da qualidade do serviço de IB. 
Figura 1 - Uma proposta de integração do modelo de Kano, SERVQUAL e QFD

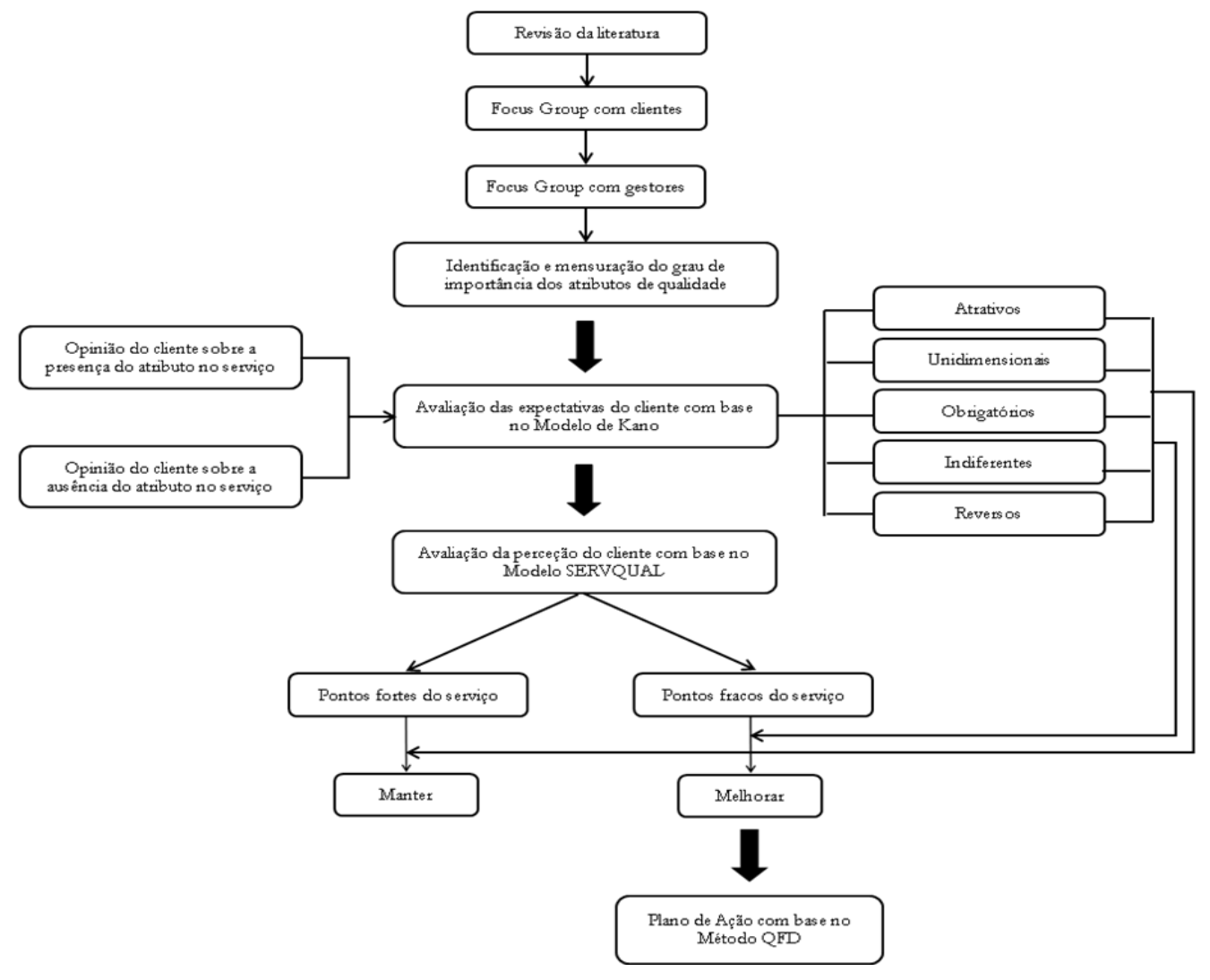

\subsection{Proposições de pesquisa}

Ao estudar os três instrumentos, verificou-se que o SERVQUAL pode ajudar a estabelecer os atributos para avaliar os serviços. Os serviços prestados via Internet devem suportar todos os processos de um serviço efetuado na forma tradicional (Santouridis et al., 2009). As dimensões originais do SERVQUAL, desenvolvidas para avaliar a qualidade dos serviços realizados dentro de um espaço físico, são úteis e aplicáveis aos serviços oferecidos via Web (Iwaarden, Wiele, Ball e Millen, 2004). Assim, sugere-se o seguinte:

Proposição 1: O serviço de IB pode ser avaliado com base nas cinco dimensões originais do SERVQUAL.

Constatou-se também que o SERVQUAL apresenta alguns problemas, sobretudo a influência mútua entre expetativas e perceções (Li, Tan e Xie, 2002). Por isso, muitos estudos têm optado por não avaliar as expetativas (Jayawardhena, 2004), avaliando só as perceções. Cronin e Taylor (1992) afirmam que medir apenas as perceções é suficiente para conhecer a qualidade do serviço. Mas, as empresas precisam de conhecer as expetativas dos clientes (Carman, 1990). O modelo de Kano permite conhecer o que os clientes desejam. Para tornar a avaliação das expetativas mais rigorosa, convém que o questionário de Kano seja aplicado a clientes que ainda não tenham utilizado o serviço e a clientes que já o utilizaram. No entanto, para medir as perceções, todas as pessoas inquiridas deverão já ter utilizado o serviço pelo menos uma vez. Pode-se, então, definir a seguinte proposição:

Proposição 2: O modelo de Kano pode ser utilizado como alternativa para identificar e classificar as expetativas dos clientes num primeiro momento, distinto daquele em que se vai avaliar as perceções.
Um outro problema do SERVQUAL é não indicar quais os atributos mais relevantes para a satisfação dos clientes (Jayawardhena, 2004). Todas as dimensões do SERVQUAL são consideradas necessárias para alcançar a excelência nos serviços, mas não são todas consideradas igualmente importantes. Como foi referido, o modelo de Kano permite ultrapassar esta limitação. $O$ objetivo desta ferramenta é categorizar os atributos dos serviços quanto à capacidade de satisfazer as necessidades dos clientes (Ardhiyani e Singgih, 2010). Assim, formula-se a seguinte proposição:

Proposição 3: As expetativas dos utilizadores do IB podem ser classificadas em cinco categorias: atrativas, unidimensionais, obrigatórias, indiferentes e reversas.

Após conhecer a "voz do cliente", é necessário descobrir como alcançar a sua satisfação e como aumentar a qualidade do serviço. Os modelos SERVQUAL e Kano são úteis para compreender as exigências dos clientes, enquanto o QFD é útil para transformar as exigências dos clientes em características técnicas do produto. Além de ser usado na produção de novos produtos, é também usado na melhoria contínua de produtos já existentes (Mohr-Jackson, 1996). O QFD ajuda os gestores na tomada de decisões acerca do desenvolvimento de um website (loannou, Pramataris e Prastacos, 2004).

Proposição 4: A aplicação do QFD fornece um plano de ação para melhorar a qualidade do serviço de IB.

Os três instrumentos a que este artigo recorre apresentam diferenças significativas entre si, nomeadamente nos objetivos que permitem atingir e na informação que disponibilizam aos gestores, mas todos oferecem contributos relevantes para a excelência dos serviços. Os modelos SERVQUAL e Kano são úteis para descobrir aquilo que o cliente quer. O QFD, por seu turno, é útil para identificar os aspetos em que se devem introduzir 
melhorias no produto/serviço. A utilização dos três instrumentos, de forma combinada, deverá permitir superar as desvantagens inerentes a cada um deles. Assim, define-se a última proposição.

Proposição 5: A integração do SERVQUAL, do modelo de Kano e do QFD contribui para uma melhoria na forma como se avalia a qualidade dos serviços.

Pelo exposto neste artigo, ficou claro que é possível combinar os três instrumentos de formas bastante diferentes. Neste artigo propõe-se uma forma distinta daquela que tem vindo a ser utilizada e defendida na literatura. Esta forma alternativa de integração dos três modelos tem como grande vantagem a possibilidade de tirar um melhor proveito das características de cada um dos modelos e também a de evitar algumas das suas desvantagens (vide Tabela 2). É essa nova forma de integrar o modelo de Kano, o SERVQUAL e o QFD que detalhamos um pouco mais na secção seguinte deste artigo.

\subsection{Proposta metodológica}

Apesar da sobrestimação frequente das taxas de insucesso de métodos e técnicas de gestão (Cândido e Santos, 2015), incluindo a sobrestimação das taxas de insucesso de ferramentas de gestão da qualidade (Cândido e Santos, 2011), considera-se importante ilustrar com algum detalhe a proposta metodológica idealizada na Secção 3.1 e ilustrada na Figura 1. Descreve-se uma forma possível de aplicação da metodologia e não a única forma possível.

Fase 1 - Identificação e importância dos atributos do serviço

A primeira fase consiste em fazer o levantamento dos principais atributos de websites bancários e avaliar o grau de importância desses atributos para o cliente. 0 conjunto destes atributos deverá abranger todas as fases do processo de compra (Li et al., 2002), designadamente, a procura de informação, a navegação no website, o pedido do cliente, a interação com o cliente, a entrega e a satisfação com a resposta ao pedido efetuado pelo cliente (Wolfinbarger e Gilly, 2003).

O Banco deve começar por fazer uma revisão da literatura sobre marketing e sobre sistemas de informação que aborde a experiência do consumidor em ambientes online. O objetivo é apurar quais os atributos referidos pela literatura. Podem também ser consultados especialistas na matéria. Em seguida, o Banco deve aplicar a metodologia de focus group, com o objetivo de estudar as dimensões da qualidade e selecionar os atributos que irão pertencer a cada dimensão. Os bancos poderiam estudar, sobretudo, as cinco dimensões do SERVQUAL, porque todas elas são aplicáveis à Internet (Santouridis et al., 2009 e Iwaarden et al., 2004). O primeiro focus group seria composto por clientes que já tenham utilizado o serviço de IB e por clientes do Banco que ainda não o experimentaram. Os clientes devem ter idades, géneros, níveis de educação e ocupações diferentes. Esta "discussão de grupo" deve permitir aos clientes falar sobre o ambiente Web do Banco, por isso, é importante que os clientes que ainda não experimentaram o serviço tenham facilidade em imaginá-lo. Depois, pode-se pedir aos clientes para comentarem a lista de atributos extraída da literatura. Ao mesmo tempo, verifica-se se surgem novas dimensões. Como referido anteriormente, não há inconvenientes em que alguns dos clientes já tenham experimentado o serviço, porque, nesta fase, pretende-se apenas descobrir os atributos. O segundo focus group é composto por gestores de IB pertencentes ao Banco prestador do serviço. Fornece-se-lhes a lista de atributos resultante do trabalho com o primeiro focus group, e pede-se-lhes para indicarem os atributos que consideram relevantes. Este processo deverá identificar todos os atributos, agrupados por dimensões da qualidade, que irão compor o instrumento de avaliação da qualidade do serviço de IB.

Fase 2 - Avaliação das expetativas do cliente

Após o levantamento dos atributos, o passo seguinte consiste em pedir ao cliente para categorizá-los, de modo a se conhecer as suas expetativas em relação ao serviço de IB. Para tal, sugere-se a construção de um questionário baseado no modelo de Kano. A primeira parte do questionário servirá para recolher dados pessoais dos inquiridos (idade, género, nível de educação e profissão), para que se possa estudar a categorização dos atributos entre diferentes grupos de clientes. A segunda parte será composta por uma questão positiva e outra negativa para cada atributo. As duas questões vêm uma logo após a outra, com o uso do "não" nas questões negativas. As questões assim redigidas e ordenadas oferecem melhores resultados do que ordenadas aleatoriamente ou sem o uso do "não" (Tontini e Theiss, 2005). Ambas as questões têm cinco opções de resposta (Eu gosto desta maneira; Deve ser desta maneira; É indiferente; Eu posso aceitar que seja desta maneira; Eu não gosto desta maneira).

O questionário é entregue a atuais e a potenciais clientes do serviço de IB. Quanto aos atuais clientes, a sua avaliação é importante, porque poderão ter criado novas expetativas no desenrolar da etapa anterior (fase 1). Quanto aos potenciais clientes, é natural que possuam expetativas distintas dos atuais clientes e é importante que tenham facilidade em imaginar o ambiente Web do Banco. Convém referir que não existe nenhum problema se alguns clientes já tiverem experimentado o serviço, porque as perceções são avaliadas na fase seguinte e, os inquiridos nessa fase não serão necessariamente os mesmos desta.

O método mais adequado para pesquisas com o modelo de Kano são as entrevistas orais (Sauerwein et al., 1996). Assim, o questionário seria entregue pessoalmente aos participantes e aplicado por meio de entrevista.

\section{Fase 3 - Avaliação das perceções do cliente}

Depois de medir as expetativas dos clientes do serviço de IB, sugere-se a realização de um outro estudo para avaliar o grau de satisfação com o serviço. Neste sentido, deverá ser elaborado um questionário a partir do SERVQUAL, mas apenas com a parte correspondente às perceções. A parte que mede as expetativas não será necessária porque, como se viu neste artigo, propõe-se a sua substituição pela análise efetuada na fase 2 .

O questionário para avaliação das perceções pode ser dividido em duas partes. A primeira serve para recolher dados pessoais dos clientes: idade, género, nível de educação, profissão e frequência com que utiliza o serviço de IB. A segunda serve para medir o grau de satisfação dos clientes com cada atributo identificado na fase 1 . Pode utilizar-se uma Escala de Likert de cinco pontos (discordo totalmente; discordo parcialmente; sem opinião; concordo parcialmente; concordo totalmente), visto estas escalas terem rótulo nas opções de resposta, o que facilita a resposta e a análise dos resultados. 
Os inquiridos terão que ser utilizadores atuais do serviço e, na sua maioria, deverão ter uma conta no Banco que pretende fazer a avaliação e outra conta no Banco principal concorrente.

A informação recolhida desta forma é útil para a avaliação da concorrência na aplicação do QFD. Além disso, os clientes inquiridos deverão ter um perfil variado para se poder conhecer a perceção em diferentes segmentos.

Tratando-se de um serviço online, sugere-se o envio do questionário aos clientes por e-mail. $\mathrm{O}$ e-mail é o método mais favorável para pesquisas de satisfação do cliente (Sauerwein et al., 1996), pois tem baixo custo (Sauerwein et al., 1996) e uma taxa de resposta elevada (Jayawardhena, 2004).

Fase 4 - Aplicação do QFD

A quarta e última fase consiste em aplicar o QFD. O objetivo é saber quais os aspetos que precisam de ser melhorados no serviço de IB. A partir da "voz do cliente", ouvida nas fases 2 e 3, constróise a "Casa da Qualidade", cuja finalidade é transformar os requisitos do cliente em requisitos técnicos para o IB. Para ajudar a estabelecer os requisitos técnicos de um website, propõe-se a realização de entrevistas a especialistas em web design. Os web designers devem trabalhar em conjunto com outros departamentos da empresa. As equipas de marketing, de engenharia e de produção devem trabalhar juntas para que os produtos/serviços reflitam os desejos dos clientes (Hauser e Clausing, 1988). No fim deste processo, os bancos terão um plano de ação para melhorar a qualidade dos seus serviços de IB.

A concluir, a Tabela 3 apresenta uma síntese do contributo teórico deste artigo, com uma comparação entre a forma como os três modelos têm sido integrados até aqui na literatura e a forma como se propõe neste artigo que os três passem a ser utilizados em conjunto.

Tabela 3 - Sumário da integração dos três instrumentos sugerida na literatura e a sugerida neste artigo

\begin{tabular}{|c|c|c|c|}
\hline \multicolumn{4}{|c|}{ INTEGRAÇÃO DOS TRÊS INSTRUMENTOS SUGERIDA NA LITERATURA EXISTENTE } \\
\hline & SERVQUAL & KANO & QFD \\
\hline \multicolumn{4}{|c|}{ INTEGRAÇÃO DOS TRÊS INSTRUMENTOS SUGERIDA NESTE ARTIGO } \\
\hline & KANO & SERVQUAL & QFD \\
\hline $\begin{array}{l}\text { Objetivos da } \\
\text { aplicação na ordem } \\
\text { sugerida }\end{array}$ & $\begin{array}{l}\text { Conhecer as expetativas dos clientes de Internet } \\
\text { Banking. }\end{array}$ & $\begin{array}{l}\text { Conhecer as perceções dos } \\
\text { clientes de Internet Banking. }\end{array}$ & $\begin{array}{l}\text { Definir quais os aspetos que } \\
\text { precisam de ser melhorados no } \\
\text { serviço de Internet Banking. }\end{array}$ \\
\hline $\begin{array}{l}\text { Recomendações } \\
\text { para a correta } \\
\text { aplicação na ordem } \\
\text { sugerida }\end{array}$ & $\begin{array}{l}\text { Elaborar um questionário composto por duas partes: } \\
\text { a primeira parte servirá para recolher dados pessoais } \\
\text { dos inquiridos; a segunda parte será composta por } \\
\text { uma questão positiva e outra negativa para cada } \\
\text { atributo. } \\
\text { Sugere-se que as duas questões de cada par sejam } \\
\text { apresentadas uma imediatamente após a outra e o } \\
\text { uso do "Não" nas questões negativas. } \\
\text { Sugere-se também que as questões tenham cinco } \\
\text { opções de resposta. }\end{array}$ & $\begin{array}{l}\text { Elaborar um questionário a } \\
\text { partir do SERVQUAL, mas } \\
\text { apenas com a parte } \\
\text { correspondente às perceções. } \\
\text { A primeira parte do } \\
\text { questionário servirá para } \\
\text { recolher dados pessoais dos } \\
\text { inquiridos; a segunda parte } \\
\text { servirá para medir o grau de } \\
\text { satisfação dos clientes com } \\
\text { cada atributo. } \\
\text { Sugere-se utilizar uma Escala } \\
\text { de Likert de cinco pontos. }\end{array}$ & $\begin{array}{l}\text { Construir uma "Casa da } \\
\text { Qualidade". }\end{array}$ \\
\hline $\begin{array}{l}\text { Benefícios a retirar } \\
\text { da aplicação na } \\
\text { ordem sugerida }\end{array}$ & $\begin{array}{l}\text { Servir como alternativa à secção das expetativas do } \\
\text { SERVQUAL. } \\
\text { Ao contrário do SERVQUAL, que considera todos os } \\
\text { atributos igualmente importantes, o modelo de Kano } \\
\text { categoriza os atributos do serviço. }\end{array}$ & $\begin{array}{l}\text { A satisfação dos clientes não é } \\
\text { avaliada com base na diferença } \\
\text { entre perceção e expectativa. }\end{array}$ & $\begin{array}{l}\text { Chegar a um plano de ação para } \\
\text { melhorar a qualidade do serviço } \\
\text { de Internet Banking. }\end{array}$ \\
\hline $\begin{array}{l}\text { Erros a evitar na } \\
\text { aplicação na ordem } \\
\text { sugerida }\end{array}$ & $\begin{array}{l}\text { Aplicar o questionário de Kano conjuntamente com o } \\
\text { questionário SERVQUAL. }\end{array}$ & $\begin{array}{l}\text { Aplicar as duas secções do } \\
\text { questionário SERVQUAL, uma } \\
\text { vez que iria resultar numa } \\
\text { duplicação de esforços para } \\
\text { avaliar as expetativas dos } \\
\text { clientes. }\end{array}$ & $\begin{array}{l}\text { Não compreender corretamente } \\
\text { o que os clientes desejam. }\end{array}$ \\
\hline
\end{tabular}




\section{Conclusão}

A qualidade dos serviços de IB tornou-se muito importante para a competitividade dos bancos. As instituições financeiras estão a procurar métodos para prestar serviços com alta qualidade que cumpram rigorosamente as necessidades ou as preferências dos seus clientes (Yu, 2008).

Baseado na revisão da literatura e numa análise crítica aos estudos já elaborados sobre o tema, este artigo centrou-se no desenvolvimento teórico de um modelo de integração do SERVQUAL, do modelo de Kano e do QFD, que é diferente daqueles apresentados na literatura. Com o modelo proposto, constituído por quatro etapas, espera-se contribuir para um melhor entendimento da "voz do cliente", uma vez que o resultado final da avaliação não é baseado na diferença entre expectativa e perceção e não enferma de um conjunto de outras desvantagens identificadas neste estudo, contribuindo assim para a melhoria da qualidade dos serviços de IB.

Trabalhos futuros poderão estudar a forma como os bancos implementam o modelo aqui proposto, a fim de testar a sua confiabilidade e validade. Poderão também estudar a forma como os bancos implementam outros modelos que integrem os três instrumentos, pois defende-se que existem outras formas alternativas de o fazer. Trabalhos futuros poderão ainda estudar a aplicabilidade do modelo proposto neste artigo (Figura 1) a outros tipos de serviços. $\mathrm{O}$ artigo apresenta um conjunto de proposições teóricas em que assenta o modelo, as quais carecem de investigação futura que lhes dê suporte com base em evidência empírica.

\section{Agradecimentos}

Os autores agradecem as sugestões de dois revisores anónimos e dos editores que muito contribuíram para melhorar este artigo. Este trabalho foi financiado por Fundos FEDER através do Programa Operacional Fatores de Competitividade - COMPETE (POCI-01-0145-FEDER-007659) e por Fundos Nacionais através da FCT - Fundação para a Ciência e a Tecnologia no âmbito do projeto UID/ECO/04007/2013.

\section{Referências}

Akao, Y. (1972). New product development and quality assurance - quality deployment system. Standardization and Quality Control, 25(4), 7-14.

Akao, Y. (1990). Quality function deployment: Integrating customer requirements into product design. Cambridge: Productivity Press.

Akinci, S., Atilgan-Inan, E. e Aksoy, S. (2010). Reassessment of E-S-Qual and ERecS-Qual in a pure service setting. Journal of Business Research, 63, 232-240.

Angelakopoulos, G. e Mihiotis, A. (2011). E-banking: challenges and opportunities in the Greek banking sector. Electronic Commerce Research, 123.

Ardhiyani, N. e Singgih, M.L. (2010). Integrating SERVQUAL with Kano into quality function deployment (QFD) for better Quality of Services. Surabaya: Institut Teknologi Sepuluh Nopember.

Bouchereau, V. e Rowlands H. (1999). Analytical approaches to QFD. Manufacturing Engineering, 78(6), 249-254.

Brochand, B., Lendrevie, J., Rodrigues J.V. e Dionísio P. (1999). Publicitor. Lisboa: Publicações Dom Quixote.

Cândido, C.J.F. (2003). Contrasting Portuguese and international service quality management in the Algarve hotel industry. The International Journal of Applied Management, 4(1), 37-54.
Cândido, C.J.F. (2005). Service quality strategy implementation - A model and the case of the Algarve hotel industry. Total Quality Management \& Business Excellence, 16(1), 3-14.

Cândido, C.J.F. e Morris, D.S. (2000). Charting service quality gaps. Total Quality Management, 11(4,5\&6), S463-S472.

Cândido, C.J.F. e Morris, D.S. (2001). The implications of service quality gaps for strategy implementation. Total Quality Management, 12(7\&8), 825-833.

Cândido, C.J.F. e Santos, S.P. (2011). Is TQM more difficult to implement than other transformational strategies? Total Quality Management \& Business Excellence, 22(11), 1139-1164.

Cândido, C.J.F. e Santos, S.P. (2015). Strategy implementation: What is the failure rate? Journal of Management and Organization, 21(2), 237-262.

Carman, J.M. (1990). Consumer perceptions of service quality: An assessment of the SERVQUAL dimensions. Journal of Retailing, 66(1), 33-55.

Castillo, D.V., Montecinos, R.H. e Villagran, A.O. (2007). Validación de un instrumento de medición de la calidad del servicio bancario en internet: un análisis aplicado a la ciudad de Arica. Horizontes Empresariais, 6(2), 31-48.

Cronin Jr, J.J. e Taylor, S.A. (1992). Measuring service quality: A reexamination and extention. Journal of Marketing, 56(3), 55-68.

Grönroos, C. (2007). Service management and marketing: Customer management in service competition. England: John Wiley \& Sons.

Hauser, J.R. e Clausing, D. (1988). The house of quality. Harvard Business Review, 66(3), 63-73.

Hitt, L.M. e Frei, F. (2002). Do better customers utilize electronic distribution channels? The case of PC banking. Management Science, 48(6), 732-748.

Hsu, C., Chang, T., Wang, S. e Lin, P. (2007). Integrating Kano's model into quality function deployment to facilitate decision analysis for service quality. In Proceedings of the $8^{\text {th }}$ WSEAS Internatinal Conference on Mathematics and Computers in Business and Economics 2007 (pp. 226232). Vancouver: World Scientific and Engineering Academy and Society (WSEAS).

Hutahaean, H.A. (2009). Application of service quality improvement method with integration between SERVQUAL and Kano's model approach. In International Seminar on Industrial Engineering and Management (pp. A88-A94). Bali.

Ioannou, G., Pramataris K.C. e Prastacos G.P. (2004). A quality function deployment approach to web site development: Applications for electronic retailing. Management Technology, 13(3), 1-18.

Iwaarden, J.V., Wiele, T.V.D., Ball, L. e Millen R. (2004). Perceptions about the quality of web sites: a survey amongst students at Northeastern University and Erasmus University. Information \& Management, 41(8), 947-959.

Jayawardhena, C. (2004). Measurement of service quality in internet banking: the development of an instrument. Journal of Marketing Management, 20(1-2), 185-207.

Kano, N., Seraku, N., Takahashi, F. e Tsuji, S. (1984). Attractive quality and must-be quality. Journal of the Japanese Society for Quality Control, 14(2), 39-48.

Khurana, S. (2009). Managing service quality: an empirical study on internet banking. Journal of Marketing Management, 8(3\&4), 96-113.

Kuo, C., Yuo, S. e Lu, C.Y. (2014). Integration of the Kano and QFD model in health food development: using black beans as examples. Quality \& Quantity, 48(1), 225-242.

Li, Y.N., Tan, K.C. e Xie, M. (2002). Measuring web-based service quality. Total Quality Management, 13(5), 685-700.

Liao, Z. e Cheung, M.T. (2008). Measuring consumer satisfaction in internet banking: a core framework. Communications of the ACM, 51(4), 47-51.

Löfgren, M. e Witell, L. (2005). Kano's theory of attractive quality and packaging. Quality Management Journal, 12(3), 7-20.

Loonam, M. e O'Loughlin, D. (2008). An observation analysis of e-service quality in online banking. Journal of Financial Services Marketing, 13(2), 164-178.

Madzík, P. (2016). Increasing accuracy of the Kano model - a case study. Total Quality Management \& Business Excellence, forthcoming. Published online June 3, 2016. 
Mohr-Jackson, I. (1996). Quality function deployment: A valuable marketing tool. Journal of Marketing Theory and Practice, 4(3), 60-67.

Nelson-Rowe, L., Kraft, J. e Varney, J. (2016). The ASQ global state of quality 2: research report. Milwaukee: ASQ e APQC.

Parasuraman, A., Zeithaml, V.A. e Berry, L.L. (1985). A conceptual model of service quality and its implications for future research, Journal of Marketing, 49(4), 41-50.

Parasuraman, A., Zeithaml, V.A. e Berry, L.L. (1988). SERVQUAL: a multipleitem scale for measuring consumer perceptions of service quality. Journal of Retailing, 64(1), 12-40.

Parasuraman, A., Berry, L.L. e Zeithaml, V.A. (1991). Refinement and reassessment of the SERVQUAL scale. Journal of Retailing, 67(4), 420-450.

Pawitra, T.A. e Tan, K.C. (2003). Tourist satisfaction in Singapore - A perspective from Indonesian tourists. Managing Service Quality, 13(5), 399-411.

Rahmana, A., Kamil, M., Soemantri, E. and Olim, A. (2014) Integration of SERVQUAL and Kano model into QFD to improve quality of simulationbased training on project management. International Journal of Basic and Applied Science, 2(3), 59-72.

Santouridis, I.; Trivellas, P. e Reklitis, P. (2009). Internet service quality and customer satisfaction: examining internet banking in Greece. Total Quality Management \& Business Excellence, 20(2), 223-239.

Sauerwein, E., Bailom, F., Matzler, K. e Hinterhuber, H.H. (1996). The Kano model: How to delight your customers. In International Working Seminar on Production Economics, vol. 1 (pp. 313-327). Innsbrruck.

Song, H. (2016). A critical review of Kano's wording and its impact on attribute classification: a case study of smartphone in Korea. Total Quality Management \& Business Excellence, forthcoming. Published online on March 7, 2016.

Sullivan, R.J. (2000). How has the adoption of internet banking affected performance and risk in banks? A look at internet banking in the Tenth Federal Reserve District. In Financial Industry Perspectives 2000 (pp. 1-16). Kansas City: Federal Reserve Bank of Kansas City.

Tan, K.C. e Shen, X.X. (2000). Integrating Kano's model in the planning matrix of quality function deployment. Total Quality Management, 11(8), 1141-1151.

Tan, K.C. e Pawitra, T.A. (2001). Integrating SERVQUAL and Kano's model into QFD for service excellence development. Managing Service Quality, 11(6), 418-430.

Tontini G. e Theiss, J. (2005). Estudo sobre a confiabilidade da classificação dos atributos de um serviço pelo modelo Kano de qualidade atrativa e obrigatória. Cadernos de Pós-Graduação - Administração, 4(1), 219-235.

Wolfinbarger, M. e Gilly M.C. (2003). eTailQ: dimensionalizing, measuring and predicting etail quality. Journal of Retailing, 79(3), 183-198.

Yesilada, F.A. e Yurdakul, D. (2009). Improving healthcare service quality: an application of integrating SERVQUAL and Kano model into quality function deployment. International Journal of Business Research, 9(7), 156-165.

Yu, C. (2008). Assessing and differentiating the quality of internet-based services: a case of online banking in Taiwan. The Services Industries Journal, 28(5), 581-602.

Zeithaml, V.A., Parasuraman, A. e Malhotra, A. (2002). Service quality delivery through web sites: A critical review of extant knowledge. Journal of the Academy of Marketing Science, 30(4), 362-375.

Recebido: 19.11.2015

Reenviado: 23.07.2016

Aceite: 25.07.2016 